\title{
Neuron-specific Enolase Level after the Onset of Delayed Neurological Sequelae with Acute Carbon Monoxide Intoxication
}

\author{
Sangsoo Han ${ }^{1}$, Sangun Nah ${ }^{1}$, Sungwoo Choi ${ }^{1}$, Young Hwan Lee ${ }^{1, *}$
}

${ }^{1}$ Department of Emergency Medicine Soonchunhyang University Bucheon Hospital, Bucheon, Republic of Korea

\section{*Correspondence}

zerohwani@gmail.com

(Young Hwan Lee)

\begin{abstract}
Background: Delayed neurological sequelae (DNS) is a major problem in carbon monoxide (CO) intoxication. Many other studies have showed whether serum NSE of acute phase can predict DNS in CO poisoning. However, no study has examined NSE levels after development of DNS. Therefore, we report serum NSE levels after the onset of DNS. Case presentation: We described the NSE levels in two cases of DNS. The first was a 33-year-old woman who presented with concentration problems 39 days after CO exposure. The second was a 51-year-old woman who presented with gait disturbance and urinary incontinence 23 days after $\mathrm{CO}$ exposure. Brain magnetic resonance imaging (MRI) and NSE levels were obtained in both patients. Brain MRI confirmed brain injury and the diagnosis of DNS was made. The NSE levels of the two patients were 12.51 and $16.18 \mathrm{ng} / \mathrm{mL}$, respectively. Conclusion: Although the initial NSE elevated level may be useful in the prediction of carbon monoxide induced DNS, it may be decreased to normal reference range after the onset of DNS.
\end{abstract}

Keywords

Brain injury, Carbon monoxide, Complications, Poisoning, Neuron specific enolase

\section{Introduction}

In carbon monoxide $(\mathrm{CO})$ poisoning, delayed neurological sequelae (DNS) is a major problem in which neurological deficits occur after a lucid interval of $2-40$ days [1]. The pathophysiology of DNS is unclear, but it is thought to be caused by brain damage associated with excitatory amino acid and oxidative stress [2]. DNS is characterized by neurological symptoms such as concentration problems, amnesia, gait disturbance, mutism, and peripheral neuropathy after $2-40$ days of lucid interval [3]. The prognostic factors of DNS include higher GCS, the presence of symptoms like dizziness or headache, higher serum level of Neuron-specific enolase (NSE) [4-6].

Neuron-specific enolase (NSE) is a glycolytic enzyme that is localized primarily in neuronal cytoplasm [7]. The NSE level has been widely studied as a predictive marker of neurological sequelae after head trauma, cardiac arrest, cerebral infarction, diffuse axonal injury and seizure [8-10]. Cha et al. suggested that initial NSE levels at presentation correlated with a risk of DNS in patients with acute CO poisoning [6]. However, there is no study about NSE level after the onset of DNS to our knowledge. We report NSE levels after DNS presentation for Patients with Previous CO Intoxication.

\section{Case presentations}

\subsection{Case 1}

A 33-year-old woman presented to the emergency department (ED) in a stupor after Charcoal-burning suicide with exposure of 10 hours. She was healthy person without medical problem and was a smoker. Her initial vital signs were stable, but she had had a loss of consciousness before the arrival. She was stuporous, responding only to noxious stimuli, yet not to verbal stimuli (Glasgow coma scale $[\mathrm{GCS}]$ score $=9$; E2, V2 , M5). Pupil light and vestibule-ocular reflexes were normal. She also showed intact gag reflex and the muscle tone was normal without Babinski responses on both feet. The patient presented symmetric withdrawal to noxious stimuli in all extremities (Medical Research Council [MRC] scale grade 3). Deep tendon reflexes were brisk in all extremities. Her initial carboxyhemoglobin $(\mathrm{COHb})$ was $25.3 \%$, and NSE was $22.62 \mathrm{ng} / \mathrm{mL}$ ( $\gamma$-enolase measured using Roche cobas 8000 through electrochemiluminescence immunoassay; reference range, $0-16.33 \mathrm{ng} / \mathrm{mL}$ ). Her other laboratory findings were as follows: white blood cell count $1.25 \times 10^{4} / \mathrm{uL}$; blood urea nitrogen (BUN) $15.9 \mathrm{mg} / \mathrm{dL}$; creatinine $0.9 \mathrm{mg} / \mathrm{dL}$; $\mathrm{pH}$ 7.38; $\mathrm{pCO}_{2} 30 \mathrm{mmHg}$; bicarbonate $18.2 \mathrm{mmol} / \mathrm{L}$; sodium $138 \mathrm{mmol} / \mathrm{L}$; and ethanol $4.3 \mathrm{mg} / \mathrm{dL}$. Initial brain computed tomography $(\mathrm{CT})$ showed no significant lesions. Immediate hyperbaric oxygen $\left(\mathrm{HBO}_{2}\right)$ therapy was started within 11 hours from initial exposure of $\mathrm{CO}$ and 8 hours after last exposure. During her hospital stay, she received $\mathrm{HBO}_{2}$ therapy three 


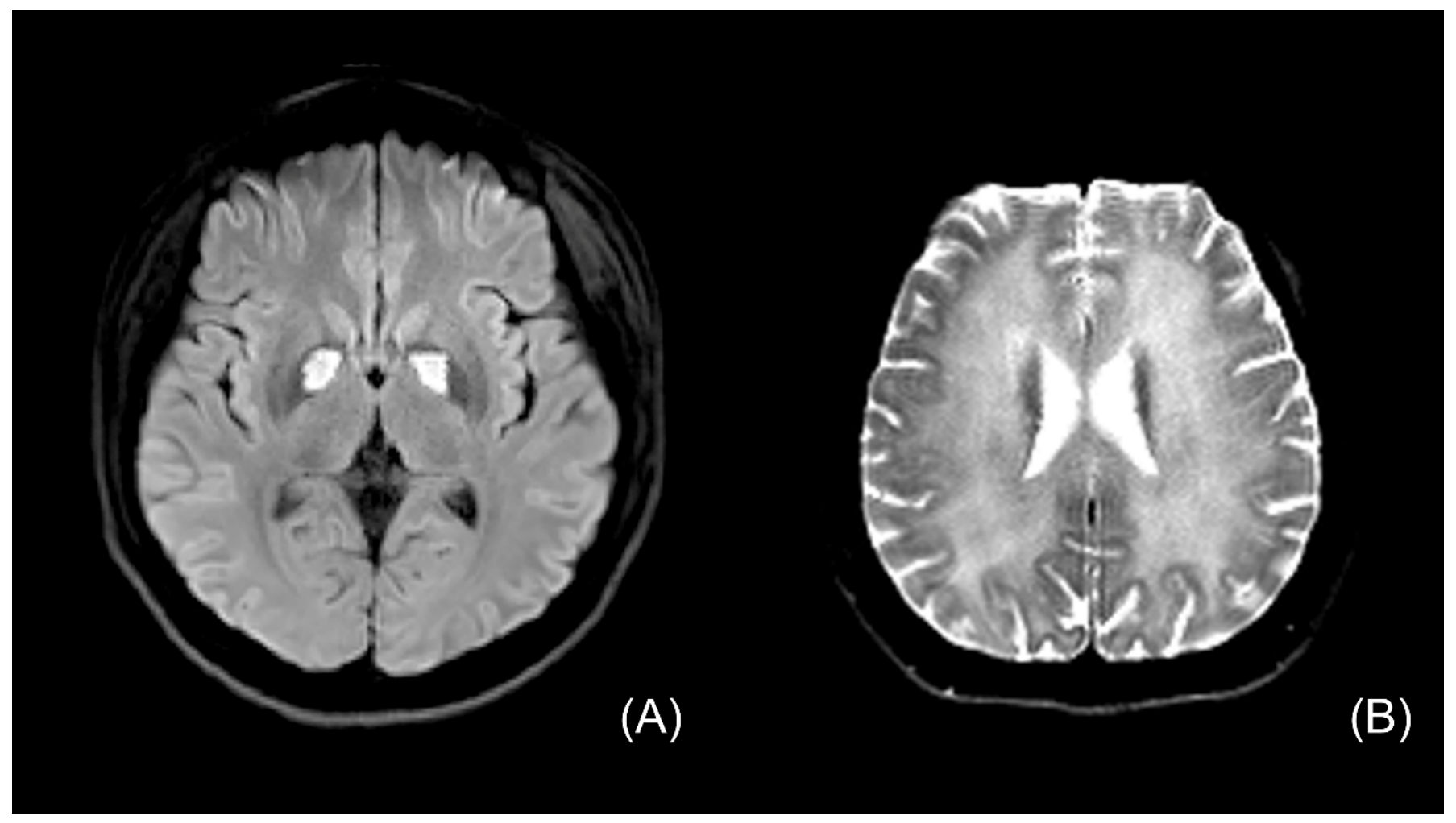

F I G U R E 1. Brain magnetic resonance imaging for case 1.

(A) diffusion-weighted imaging revealed a central high-signal intensity lesion surrounded by a low-signal-intensity ring in the globus pallidus (Eye-of-the-tiger sign) in acute carbon monoxide poisoning; (B) T2 imaging revealed extensive bilateral symmetrical confluent high-intensity cerebral white lesions after the onset of delayed neurological sequelae.

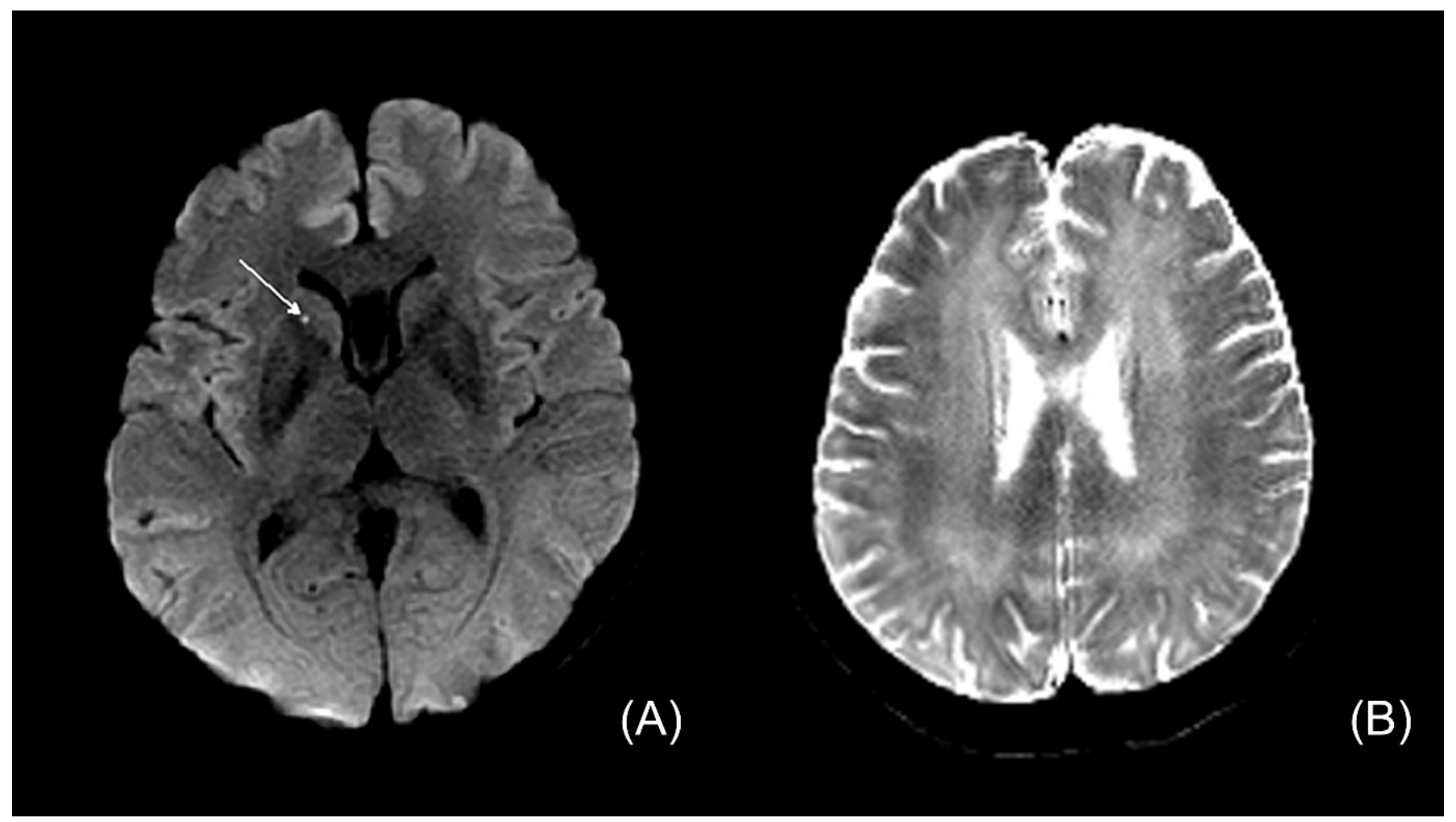

F I G U R E 2. Brain magnetic resonance imaging for case 2.

(A) diffusion-weighted imaging revealed scattered, tiny high signal intensities in the right caudate nucleus in acute carbon monoxide poisoning; (B) T2 imaging revealed apparent confluent high signal intensity in the bilateral subcortical white matter after the onset of delayed neurological sequelae. 

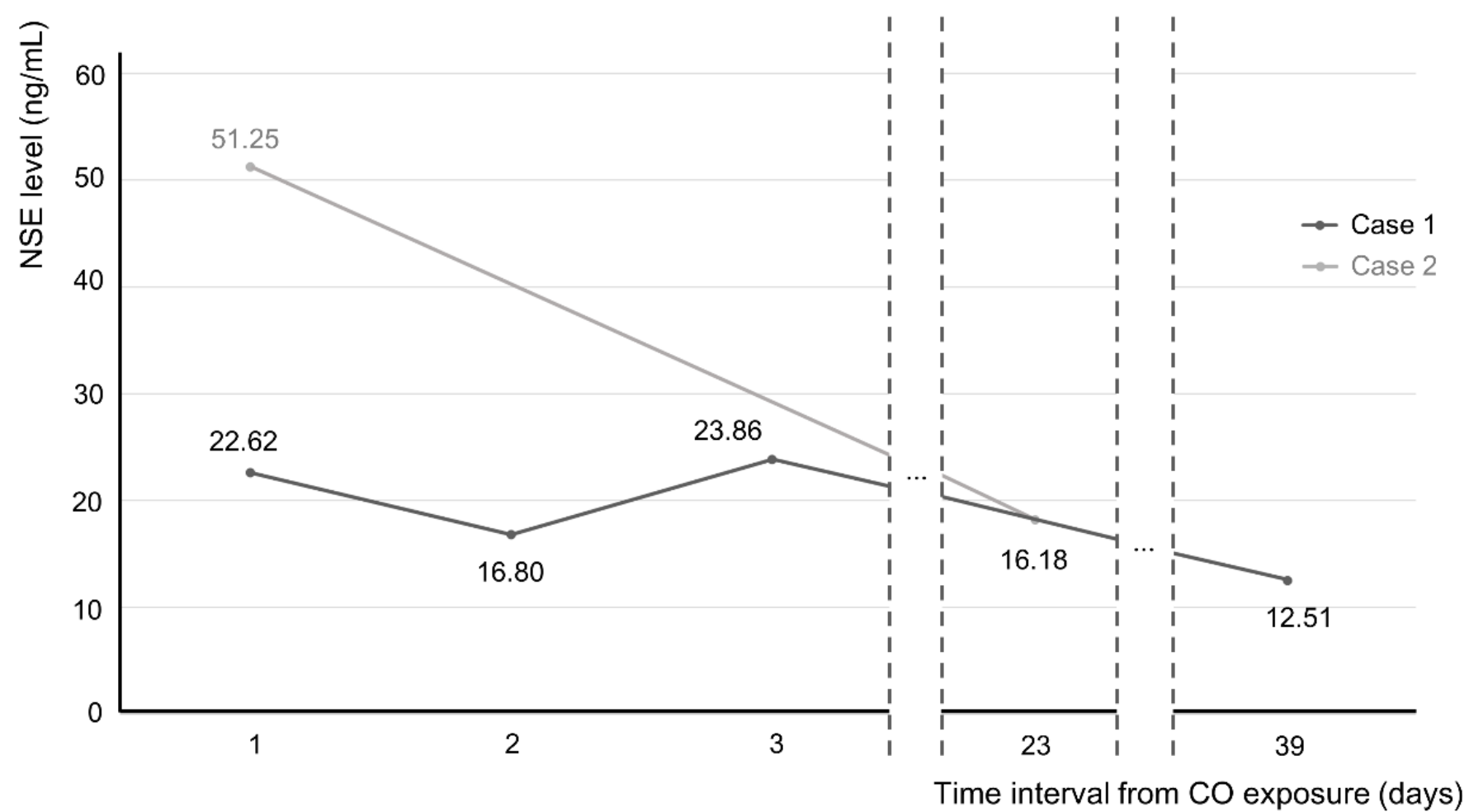

F I G U R E 3. Neuron-specific enolase (NSE) levels according to time from carbon monoxide intoxication.

In both case, NSE level was high $(22.62,51.25 \mathrm{ng} / \mathrm{mL})$ in acute carbon monoxide poisoning, and was decreased to normal range (12.51 and $16.18 \mathrm{ng} / \mathrm{mL}$ ) in delayed neurological sequelae.

times. Our hospital protocol is an initial 150-minute session at 3 atmospheres absolute (ATA), followed by two 120-minute sessions at 2 ATA, separated by $6-12$ hours, within 24 hours (IBEX MONO; IBEX medical systems Co.) [12].

After admission, she became alert without retrograde or antegrade amnesia, and her NSE level was $16.80 \mathrm{ng} / \mathrm{mL}$. Her GCS score was 15 without any neurological deficit such as motor or sensory impairment and decreased reflex. She also had no cognitive impairment like apraxia, disorientation and agnosia. On the third day of hospitalization, diffusion-weighted brain magnetic resonance imaging (MRI; Signa HDXT 3.0; GE Healthcare) revealed a central high-signal intensity lesion surrounded by a low-signal-intensity ring in the globus pallidus (Fig. 1A), and the NSE was $23.86 \mathrm{ng} / \mathrm{mL}$. She was discharged with no neurological deficits (GCS score $=15$ ) and her Mini Mental State Examination (MMSE) score was 30/30 points.

She returned to the ED with concentration problems 39 days after the $\mathrm{CO}$ exposure. Her vital signs including body temperature were within normal range. She was slightly drowsy and disoriented for time and place with GCS 13 (E4,V3, M6). Her symptoms had occurred that day. She had profound impairment in attention and short-term memory. Her MMSE score was $13 / 30$ points. The rest of neurologic and physical examination including the Kernig and Brudzinski signs were normal. Brain CT, lumbar puncture, electroencephalogram, laboratory test including ethanol and urine analysis for toxic substances were performed to rule out other possible causes for her symptoms and the tests did not show any significant abnormal findings. The serum level of NSE was $12.51 \mathrm{ng} / \mathrm{mL}$, which was in normal range. However, extensive bilateral symmetrical confluent high-intensity cerebral white lesions on brain MRI (Fig. 1B). DNS was diagnosed by a neurologist in ED. She was admitted with GCS score 13 and treated with daily $\mathrm{HBO}_{2}$ therapy for 5 weeks (37 sessions). She was transferred to a rehabilitation hospital with cognitive impairment after 5 weeks. At discharge, her MMSE score was 13/30 points with disorientation, attention deficiency and a problem in shortterm memory, and her GCS score was 13. Three months after the onset of DNS, the patient still had no improvement in symptoms.

\subsection{Case 2}

A 51-year-old woman presented to the ED in a stupor due to $\mathrm{CO}$ poisoning in a suicide attempt with Charcoal-burning. The estimated exposure time was 17 hours. She was healthy person without medical problem and was a non-smoker. Her GCS score was 10 (E2, V3, M5). Pupil light, vestibule-ocular reflexes, gag reflex and self-respiration were normal. The patient had MRC grade 3 in all extremities. Deep tendon reflexes were normal in all extremities without Babinski responses. Her initial vital signs were stable, but she had lost consciousness at home. Her initial $\mathrm{COHb}$ and $\mathrm{NSE}$ were $28.4 \%$ and 51.25 $\mathrm{ng} / \mathrm{mL}$, respectively. Her other laboratory findings were as follows: white blood cell count $1.25 \times 10^{4} / \mathrm{uL}$; BUN 21.8 $\mathrm{mg} / \mathrm{dL}$; creatinine $0.9 \mathrm{mg} / \mathrm{dL} ; \mathrm{pH}$ 7.44; $\mathrm{pCO}_{2} 36 \mathrm{mmHg}$; bicarbonate $24.5 \mathrm{mmol} / \mathrm{L}$; sodium $138 \mathrm{mmol} / \mathrm{L}$; and ethanol $2.2 \mathrm{mg} / \mathrm{dL}$. Initial brain CT showed no significant abnormal findings. She received $\mathrm{HBO}_{2}$ therapy within 18 hours from initial exposure of $\mathrm{CO}$ and 14 hours after last exposure and 
was admitted.

After admission, she became alert without amnesia and her GCS score was 15. Cognitive function such as memory, orientation, attention, and language was intact, and brain stem function such as pupillary, corneal, and gag reflex was also normal. Brain MRI showed scattered high-intensity signals in the subcortical white matter and caudate nucleus (Fig. 2A). She underwent three $\mathrm{HBO}_{2}$ sessions and was discharged on the third day of hospitalization without any neurological deficits. Her MMSE score was 30/30 points without any cognitive impairment and she had no neurologic deficit (GCS score = 15).

She returned to the ED with gait disturbance and urinary incontinence on the day these symptoms developed which was 23 days after the $\mathrm{CO}$ exposure. She was alert, and had normal vital signs including body temperature. GCS score was 12 (E4, V2, M6) with normal pupil light reflex. Her MMSE score was 11/30 with disorientation, impaired attention and deficits in short term recall memory. Physical and neurologic examination were normal with no meningeal irritation signs. We performed brain CT, lumbar puncture, electroencephalogram, laboratory test including ethanol and urine analysis for toxic substances to rule out other etiology of her symptoms. The tests did not show any significant abnormal findings and the NSE level was also normal of $16.18 \mathrm{ng} / \mathrm{mL}$. Brain MRI revealed apparent confluent high signal intensity in the bilateral subcortical white matter (Fig. 2B). A Neurologist diagnosed DNS in ED. She was admitted and underwent 14 sessions of $\mathrm{HBO}_{2}$ therapy while being hospitalized for 2 weeks. Unfortunately, her symptoms did not improve, and she was transferred to a rehabilitation hospital. Her MMSE score was 11/30 at discharge with cognitive impairment like disorientation, impaired attention and problem to recall short term memory, and her GCS score was 12 . By 5 month after the discharge, there was no improvement in symptoms yet.

\section{Discussion}

NSE is a useful biochemical marker that reflects cerebral injury, such as after cardiac arrest or cerebral infarction [11, 13]. In this study, the NSE levels after the onset of DNS were not significantly elevated.

In acute $\mathrm{CO}$ intoxication, $\mathrm{CO}$ causes hypoxic stress by reducing plasma oxyhemoglobin through production of $\mathrm{COHb}$. The $\mathrm{HBO}_{2}$ therapy, which quickly removes $\mathrm{CO}$ from the plasma, but there is controversial to prevent DNS [9]. DNS is thought to be an adaptive immune response of modified myelin basic protein with a concomitant inflammatory response [2]. DNS includes a wide spectrum of symptoms that range from mild to severe and include altered consciousness, concentration problems, cognitive disturbances, amnesia, movement disorders, urinary incontinence, and even a vegetative state [14]. The diagnosis is based on clinical features and imaging findings, such as brain MRI [14].

Many studies have examined whether NSE can predict DNS early in acute $\mathrm{CO}$ poisoning. The NSE level could be elevated through the following mechanism: $\mathrm{CO}$ exposure can cause inflammation through independent pathways, such as postischemic reperfusion injury, $\mathrm{CO}$ effects vascular endothelium, oxygen radical-mediated lipid peroxidation, and nitric oxide liberated from platelets at the time of $\mathrm{CO}$ exposure, all of which can culminate in neurologic injury [6]. However, there is controversy about the relationship between NSE level and DNS. Cha et al. [6] reported that higher NSE level is related to the development of DNS, while Moon et al. [15] argued that the NSE level is not correlated with the risk of DNS. According to one study, the NSE cut off value was $36.8 \mathrm{ng} / \mathrm{mL}$ [16], and the initial NSE values in our cases was 22.62 and $51.25 \mathrm{ng} / \mathrm{mL}$, respectively. Unlike the result of the study, DNS occurred in both cases in our study. Because NSE is a marker that reflects brain damage [13], we were wondering if the NSE levels elevated even after DNS occurred. The patients revisited to ED on the day the DNS symptoms occurred, and were immediately performed a brain MRI and NSE test. Extensive hypoxic brain lesions were identified in both cases on MRI after development of DNS. However, the NSE levels were in the normal reference range (12.51 and $16.18 \mathrm{ng} / \mathrm{mL}$ ) (Fig. 3). Our hospital uses a Roche cobas 8000 to measure NSE, and our normal laboratory range is 0 to $16.33 \mathrm{ng} / \mathrm{mL}$.

DNS result from both apoptosis of neurons and astrocytes and inflammation in the brain [17]. NSE, a highly specific marker for neurons, can quantify the degree of neuronal injury, but not the overall extent of brain damage, such as astrocyte injury [7]. Therefore, the elevated NSE levels in acute CO poisoning may not be present during DNS. This is the first report about NSE levels after the onset of DNS.

There are two limitations to this report. First, though we did not use IV contrast injection during MRI because the patients didn't show any sign of infection or meningeal irritation, MRI with contrast could have been helpful to determine other possible causes like inflammation. Second, since the diagnostic criteria of DNS is not established properly, the diagnosis of DNS is primarily made on the basis of the clinical features and radiological findings of CT and conventional MRI [18]. In our case also, the diagnosis was made after excluding other possible causes through various tests first, then considering the patients' clinical manifestations and brain MRI next. And like the criteria of DNS, there is no validated scale also, so we assessed the severity of DNS with MMSE. Further research is needed to overcome these limitations.

\section{Conclusion}

Although the initial NSE elevated level may be useful in the prediction of carbon monoxide induced DNS, it may be decreased to normal reference range after the onset of DNS. Therefore, it cannot be relied upon to follow up the development of DNS. Further prospective studies should be conducted to determine the optimum duration of NSE analysis for prediction of DNS.

\section{ACKNOWLEDGMENTS}

This work was supported by the Soonchunhyang University Research Fund. 


\section{CONFLICT OF INTEREST}

The authors declare that there are no conflicts of interest.

\section{ETHICS STATEMENT}

The study protocol was approved by the Institutional Review Board of Soonchunhyang University Bucheon Hospital.

\section{FUNDING}

The authors received no financial support for the research, authorship, and/or publication of this article.

\section{AVAILABILITY OF DATA AND MATERIAL}

Please contact author for data requests.

\section{REFERENCES}

[1] Thom SR, Taber RL, Mendiguren II, et al. Delayed neuropsychologic sequelae after carbon monoxide poisoning: prevention by treatment with hyperbaric oxygen. Ann Emerg Med. 1995;25:474-480.

[2] Thom SR, Bhopale VM, Fisher D, et al. Delayed neuropathology after carbon monoxide poisoning is immune-mediated. Proc Natl Acad Sci U S A. 2004;101:13660-13665.

[3] Norris JC, Trench J, Hook R. Delayed carbon monoxide encephalopathy: clinical and research implications. J Clin Psychiatry. 1982;43:294-295.

[4] Pan K-T, Shen C-H, Lin F-G, et al. Prognostic factors of carbon monoxide poisoning in Taiwan: a retrospective observational study. BMJ open. 2019;9:e031135.

[5] Annane D, Chevret S, Jars-Guincestre M, et al. Prognostic factors in unintentional mild carbon monoxide poisoning. Intensive Care Med. 2001;27:1776-1781.

[6] Cha Y, Kim H, Do H, et al. Serum neuron-specific enolase as an early predictor of delayed neuropsychiatric sequelae in patients with acute carbon monoxide poisoning. Hum Exp Toxicol. 2018;37:240-246.

[7] P E Vos, K J B Lamers, J C M Hendriks, et al. Glial and neuronal proteins in serum predict outcome after severe traumatic brain injury. Neurology. 2004;62:1303-1310.
[8] Wiberg S, Hassager C, Stammet P, et al. Single versus serial measurements of neuron-specific enolase and prediction of poor neurological outcome in persistently unconscious patients after out-of-hospital cardiac arrest-a TTM-trial substudy. PLoS One. 2017;12:e0168894.

[9] Zaheer S, Beg M, Rizvi I, et al. Correlation between serum neuron specific enolase and functional neurological outcome in patients of acute ischemic stroke. Ann Indian Acad Neurol. 2013;16:504.

[10] Meynaar IA, Oudemans-van Straaten HM, van der Wetering J, et al. Serum neuron-specific enolase predicts outcome in post-anoxic coma: a prospective cohort study. Intensive Care Med. 2003;29:189-195.

[11] Cakir Z, Aslan S, Umudum Z, et al. S-100ß and neuron-specific enolase levels in carbon monoxide-related brain injury. Am J Emerg Med. 2010;28:61-67.

[12] Weaver LK, Hopkins RO, Chan KJ, et al. Hyperbaric oxygen for acute carbon monoxide poisoning. N Engl J Med. 2002;347:1057-1067.

[13] Rosen H, Sunnerhagen KS, Herlitz J, et al. Serum levels of the brainderived proteins S-100 and NSE predict long-term outcome after cardiac arrest. Resuscitation. 2001;49:183-191.

[14] Gilmer B, Kilkenny J, Tomaszewski C, et al. Hyperbaric oxygen does not prevent neurologic sequelae after carbon monoxide poisoning. Acad Emerg Med. 2002;9:1-8.

[15] Moon J, Chun B, Lee S, Jung E. Serum neuron-specific enolase levels at presentation and long-term neurological sequelae after acute charcoal burning-induced carbon monoxide poisoning. Clin Toxicol. 2018;56:751758.

[16] Jung JW, Lee JH. Serum lactate as a predictor of neurologic outcome in ED patients with acute carbon monoxide poisoning. Am J Emerg Med. 2019;37:823-827.

[17] Jurič DM, Finderle Ž, Šuput D, et al. The effectiveness of oxygen therapy in carbon monoxide poisoning is pressure- and time-dependent: a study on cultured astrocytes. Toxicol Lett. 2015;233:16-23.

[18] Gilmer B, Kilkenny J, Tomaszewski C, et al. Hyperbaric oxygen does not prevent neurologic sequelae after carbon monoxide poisoning. Acad Emerg Med. 2002;9:1-8.

How to cite this article: Sangsoo Han, Sangun Nah, Sungwoo Choi, Young Hwan Lee. Neuron-specific Enolase Level after the Onset of Delayed Neurological Sequelae with Acute Carbon Monoxide Intoxication. Signa Vitae. 2021;17(1):200-204. doi:10.22514/sv.2020.16.0042. 